\title{
Prevention of disease progression in a patient with a gastric cancer-re-recurrence. Outcome after intravenous treatment with the novel antineoplastic agent taurolidine. Report of a case Chris Braumann*1,2, Goetz Winkler ${ }^{1}$, Patrick Rogalla ${ }^{3}$, Charalambos Menenakos ${ }^{1}$ and Christoph A Jacobi ${ }^{1}$
}

Address: ${ }^{1}$ Department of General, Visceral, Vascular and Thoracic Surgery, Medical Faculty Charité, Humboldt University, Berlin, Germany, ${ }^{2}$ Division of Molecular Biology, Medical Faculty Charité, Humboldt University, Berlin, Germany and ${ }^{3}$ Department of Radiology, Medical Faculty Charité, Humboldt University, Berlin, Germany

Email: Chris Braumann* - chris.braumann@charite.de; Goetz Winkler - g1winkler@online.de; Patrick Rogalla - patrick.rogalla@charite.de; Charalambos Menenakos - menenakos@hotmail.com; Christoph A Jacobi - christoph.jacobi@charite.de

* Corresponding author

\section{Published: 24 June 2006}

World Journal of Surgical Oncology 2006, 4:34 doi:10.1 186/1477-78/9-4-34

This article is available from: http://www.wjso.com/content/4/I/34

(c) 2006 Braumann et al; licensee BioMed Central Ltd.

This is an Open Access article distributed under the terms of the Creative Commons Attribution License (http://creativecommons.org/licenses/by/2.0), which permits unrestricted use, distribution, and reproduction in any medium, provided the original work is properly cited.
Received: 28 November 2005

Accepted: 24 June 2006

\begin{abstract}
Background: Taurolidine (TRD) is a novel agent with multimodal antineoplastic effects. We present the case of a tumor remission after intravenous administration of taurolidine in a patient with gastric cancer re-recurrence.

Case presentation: A 58 years old male patient suffering from a gastric adenocarcinoma was submitted to partial gastrectomy and partial liver resection (pT2, PNI, PMI (liver segment 2), N0, V0). 24 months later a local recurrence was diagnosed and the patient was reoperated. Postoperatively the patient underwent a palliative chemotherapy with eloxatin, FU, and leucovorin. A subsequent CT-revealed a liver metastasis and a recurrence adjacent to the hepatic artery. After successful radiofrequency ablation of the liver metastasis the patient was intravenously treated with $2 \%$ taurolidine. The patient endured the therapy well and no toxicity was observed. CT-scans revealed a stable disease without a tumor progression or metastatic spread. After 39 cycles the patient was submitted to left nephrectomy due to primary urothelial carcinoma and died 2 days later due to myocardial infarction. Postmortem histology of the esophageal-jejunal anastomosis and liver revealed complete remission of the known metastasized gastric adenocarcinoma.
\end{abstract}

Conclusion: The intravenous treatment with $2 \%$ taurolidine led to a histological remission of the tumor growth without any toxicity for the patient.

\section{Background}

Surgical resection remains the only treatment modality offering the possibility of cure for patients with gastric cancer. Nevertheless, the extent of resection required for potentially curative operations remains controversial. Opposite to japanese studies (D3 and D4 lymph node dis- section) [1], different trials in Europe and USA showed significantly lower morbidity and mortality rates with no survival difference when a less radical lymph node dissection of perigastric- (D1) or regional lymph nodes outside the perigastric area (D2) was performed [2-4]. So far, adjuvant chemotherapy in gastric cancer has failed to improve 
survival (e.g. 5-fluorouracil (5-FU) plus high dose methotrexate plus doxorubicin (FAMTX), etoposid plus cisplatin (EAP), etoposide plus leucovorin plus 5-FU (ELF), and epirubicin plus cisplatin plus 5-FU (ECF) $[5,6]$. In a locally advanced gastric cancer or in case of relapse the prognosis is very poor. Therefore, new chemotherapeutic agents are tested in order to improve survival and the quality of life.

The antineoplastic substance taurolidine was found to suppress intraperitoneal gastrointestinal tumor growth after laparotomy and laparoscopy in animals [6,7]. A single intravenous injection of $0,5 \%$ taurolidine did not affect tumor growth in rats $[8,9]$. Unpublished data showed a reduction of advanced intraperitoneal neoplasm after an intravenous long-term treatment (unpublished). Taurolidine induces apoptosis and inhibited tumor growth in various cells lines in vitro [10-13]. The agent was found to decrease the production of Tumor Necrosis Factor alpha (TNF $\alpha$ ) and Interleukin-1 $\beta$ (IL-1 $\beta$ ) in peritoneal macrophages [14] as well as the TNF $\alpha$ and the VEGF secretion by gastrointestinal neoplasms [15].

Based on these data, a male patient with a gastric cancer re-relapse was palliatively treated with this agent, undergoing 42 cycles of a 7-days'-intravenously treatment. The effects on relevant blood parameters, tumor markers, side effects, and tumor size were analyzed.

\section{Case presentation}

A 58-year-old man (83 kilo gram, kg) presented to our Surgical Department because of a gastric cancer eight years ago. The past medical history was significant for higher PSA levels for 10 years without clinical relevance, an acute myocardial infarction which was treated with coronary arteries' stenting and an arterial occlusive disease (Fontaine IIb). The patient was submitted to surgery and a subtotal gastrectomy with a gastrojejunostomy and a partial

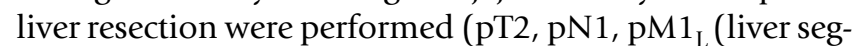
ment 2), N0, V0). No adjuvant chemotherapy was carried out.

During the follow-up a local recurrence has been detected in the abdominal CT-scan 24 months after surgery. The patient was then submitted to a total gastrectomy with Roux en Y esophagojejunostomy. A palliative chemotherapy (eloxatin, 5-fluoruracil, leukovorin) was performed for 10 months but no remission was observed. Twelve months later the CT-scan revealed a stable tumor disease despite chemotherapy adjacent to the hepatic artery. Then the patient underwent a palliative intravenous therapy with $2 \%$ taurolidine for seven days (per months) with a daily dose of $300 \mathrm{mg}$ per $\mathrm{kg}$ body weight. After twelve cycles the CT-scan revealed a tumor mass reduction and the therapy was terminated. Three months later the patient experienced abdominal discomfort. Diagnostics showed one liver metastasis in segment 8 which was successfully treated with radiofrequency ablation (Figure 2). The patient was treated with taurolidine monthly ever since. After the chemotherapy no. 32 an additional primary urothelial carcinoma in two different sites of the urinary tract (left kidney, urinary bladder) The lesion in the bladder was localy excized (pTa, G2) while the kidney lesion was put under observation by the urologists. After treatment with a total of 39 cycles with taurolidine the patient was in a good clinical condition and showed no life quality deficit. At this time the CT-scan (Figure 3) showed no tumor progression next to the hepatic artery (stable disease). Four weeks after the completion of the treatment the patient was admitted to the urology department of our hospital in order to be submitted to surgical treatment of his urothelial carcinoma. A left nephrectomy was performed. Although there were no intraoperative problems the patient unexpectedly died of an acute myocardial infarct 48 hours after surgery.

Post mortem histology of the esophageal-jejunal anastomosis and liver revealed surprisingly no signs of the known adenocarcinoma. We thought this finding was extremely interesting as we had actually been in front of a complete remission of his gastrointestinal tumor.

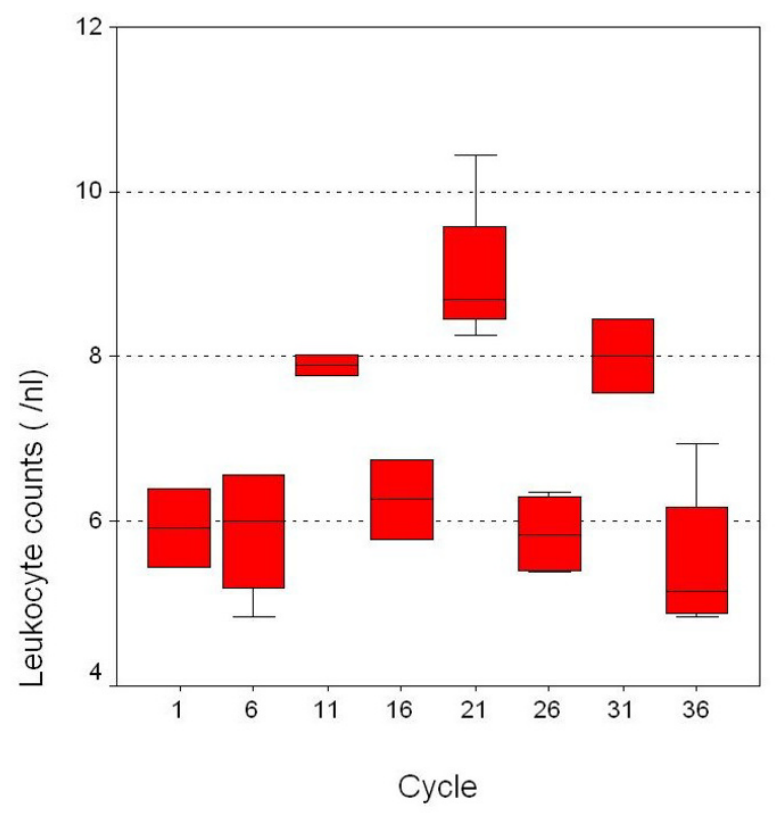

Figure I

Peripheral leukocyte counts during therapy (values of every fifth cycle is shown). 


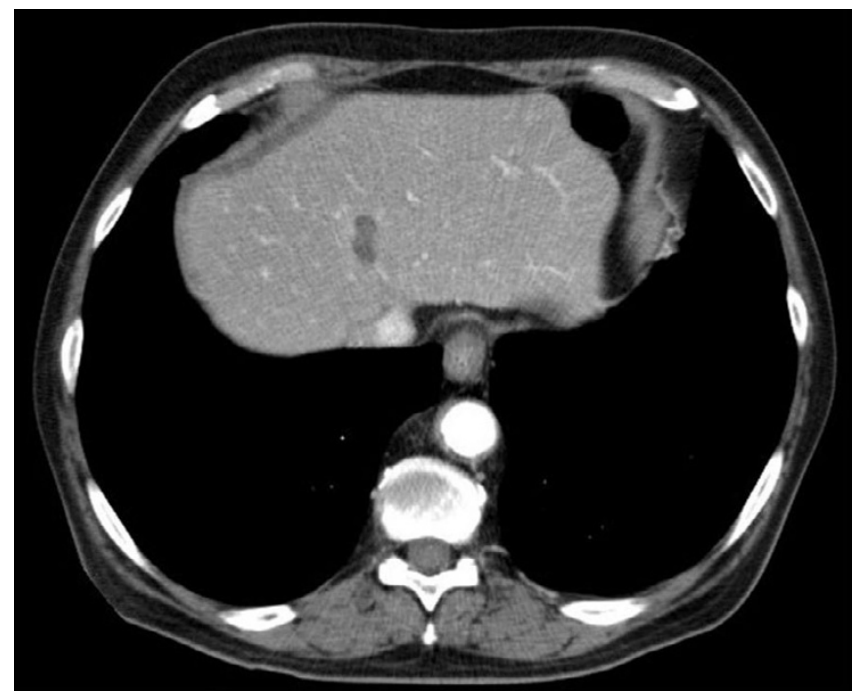

Figure 2

Region of thermoablation, liver segment 8.

\section{Methods and materials}

The antineoplastic agent taurolidine was intravenously applied in a $2 \%$ solution (Geistlich Pharma AG Wohlhusen, Switzerland) using a port catheter system. The monthly therapy $(300 \mathrm{mg} / \mathrm{kg} /$ day according 24,9 g taurolidine/day) was given for 5 days in four hours' therapy sessions and 2 hours' intervals. Tumor markers (carcino embryonal antigen-CEA, CA 72-4) were determined at the beginning and at the end of the therapies. Blood counts

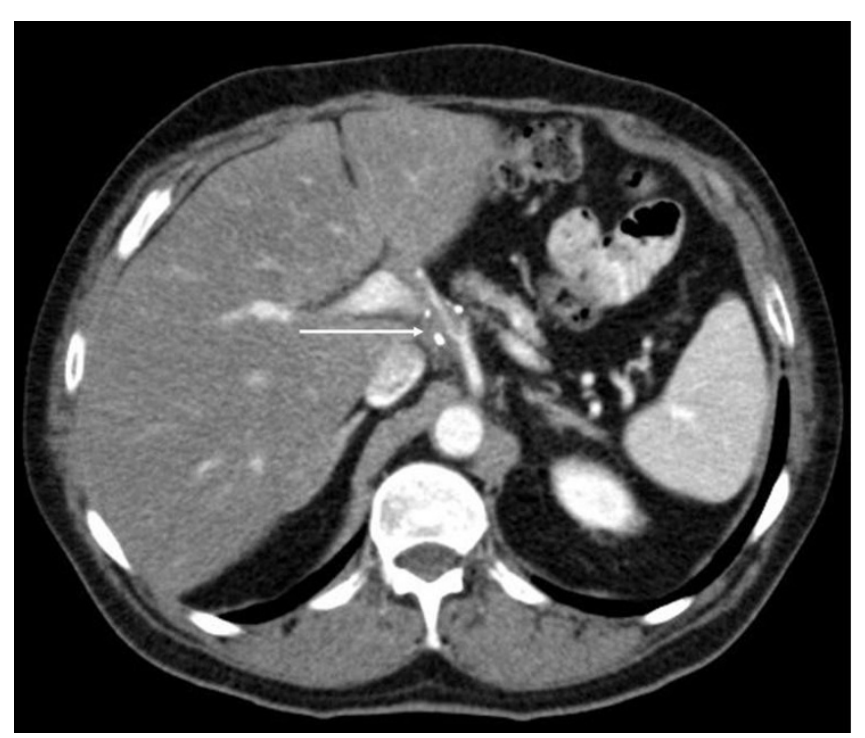

Figure 3

A stable disease of the gastric carcinoma surrounding the hepatic artery (arrow) could be detected after the 36 cycles chemotherapy (clips). and biochemistry were examined every day. Disease progression was radiologically followed-up with an abdominal CT-scan every two months.

Quality of life was assessed using the EORTC QLQ-C30 questionnaire [16], a 30-questionnaire score composed of multi-item and single-items scales reflecting the quality of life multidimensionally. It incorporates five functional scales (physical, role, cognitive, emotional, social), three symptom scales (fatigue, pain, nausea, vomiting) and a global health and quality-of life scale. The remaining single items evaluate additional symptoms commonly reported by cancer patients as well as the perceived financial impact of the disease and treatment.

GCP guidelines and Helsinki declaration for studies on humans were complied with. The Ethics Committee of the Charité Campus Mitte, University Hospital, Universitaetsmedizin Berlin, Germany, approved the study design.

\section{Results}

The patient tolerated the therapy sessions well. As far as toxicity is concerned, we have to emphasize on the fact, that the administered taurolidine concentration (2\%) did not impair the leukopoiesis or the patient's quality of life (Table 1 and 2). Tumor markers, conjugated bilirubine, creatinine or urea did not increase. Additionally the agent infusion is electrolytes-free no other side-effects from taurolidine treatment in the blood parameters were observed. Low toxicity is a main beneficial feature of taurolidine, which substantially differentiates the substance from traditional chemotherapeutic agents, which are quite often related to devastating toxic complications. According to standardized clinical and CT-scan criteria with consecutive comparisons of the CT scans during the taurolidine treatment, we were able to detect no progression of the tumor growth, so the disease was classified as stable. Moreover, it could be shown that after radiofrequency ablation liver tissue regenerates again. It has to be pointed out, that although consecutive CT-scans showed stable disease, the post mortem histological examination of the esophageal-jejunal anastomosis and liver surprisingly confirmed the absence of any residual disease.

\section{Discussion}

The incidence of gastric cancer has decreased worldwide. Despite this decrease, gastric cancer remains an important surgical topic, as much as its epidemiology is changing, thus making therapy for the disease more demanding. At this time, surgery is the only potentially curative therapy for this malignancy $[2,4,17,18]$. The aim of any surgical approach to gastric carcinoma should be complete resection with no residual tumor left behind at the end of the operation (R0 resection). Nevertheless, radical surgery after relapse is often extremely difficult - or even impossi- 
Table I: The intravenous taurolidine treatment did not impair the above mentioned blood parameters after 39 cycles (Wilcoxon test, $p=$ not significant).

\begin{tabular}{|c|c|c|}
\hline Serum parameters & Median & Range \\
\hline CA $72-4$ (tumor marker, $\mathrm{U} / \mathrm{ml}$ ) & 0.6 & $0.3-1.8$ \\
\hline CEA (tumor marker, $\mu \mathrm{g} / \mathrm{l}$ ) & 18.8 & $6.7-32.4$ \\
\hline Leukocyte $(/ \mu \mathrm{l})$ & 6.4 & $4.8-13.0$ \\
\hline Hemoglobin $(\mathrm{g} / \mathrm{l})$ & 13.0 & $10.4-14.8$ \\
\hline Hemocrit (\%) & 40 & $32-44$ \\
\hline Thrombocyte $\left(10^{3} / \mu \mathrm{l}\right)$ & 225 & $|7|-32 \mid$ \\
\hline Glucose (mg/dl) & 94 & $54-283$ \\
\hline Saline $(m g / d l)$ & 138 & $130-149$ \\
\hline Potassium (mg/dl) & 4.3 & $3.6-5.3$ \\
\hline Creatinine (mg/dl) & I.I & $0.74-1.75$ \\
\hline Urea $(\mathrm{mg} / \mathrm{dl})$ & 34 & $22-72$ \\
\hline Protein $(g / d l)$ & 7.2 & $6.1-7.4$ \\
\hline GOT (U/I) & 23 & $7-95$ \\
\hline GPT (U/l) & 13 & $8-73$ \\
\hline Total bilirubine $(\mathrm{mg} / \mathrm{dl})$ & 0.3 & $0.1-0.6$ \\
\hline Alkaline phosphatase (U/l) & 70 & $56-122$ \\
\hline Amylase (U/I) & 53 & $44-104$ \\
\hline Lipase (U/I) & 17.5 & $17-18$ \\
\hline INR & I.I & $1.0-1.2$ \\
\hline
\end{tabular}

ble [1] - and a standardized chemotherapy does not exist. A beneficial effect of adjuvant therapy is currently controversial. A meta analysis of 11 published trials including 2096 patients showed no significant benefit for patients who had adjuvant therapy after R0 resection [19]. Opposite to the western world, several studies clearly favoring adjuvant therapy in patients with gastric cancer have been reported from Japan, where adjuvant therapy is considered a standard modality and is initiated immediately during the postoperative period or even intraoperatively [20].

The limited therapy options with traditional chemotherapeutic substances led to intensive research for new antineoplastic agents. Taurolidine, a synthetic product derived from the aminosulfone acid taurine, consists of two aromatic rings, which are connected with a $\mathrm{CH}_{2}$-group; $\mathrm{MW}$ 284. There are several reports in the literature about the immune modulating and antineoplastic potential of this substance. Taurolidine reduced the production of TNF $\alpha$ (2 h contact, IC50 $0.5 \mathrm{mM}$ ) as well as VEGF ( 6 h contact,
IC50 $1.5 \mathrm{mM}$ ), which is a major proangiogenic factor, might indicate a possible influence on various malignancies [15]. The agent inhibited tumor growth of many entities with poor responses to current therapeutic regiments $[11,21,22]$. Dose-dependent findings are supported by McCourt et al. [7] and Calabresi et al, [10]. They showed a four-fold increase of tumor cell necrosis after treatment with increasing doses in several different malignancies in vitro. The intravenous application of taurolidine had no significant side effects at a maximum concentration of $300 \mathrm{mg}$ per kg per day.

We decided to treat our patient with taurolidine because he did not respond to the standard adjuvant iv chemotherapy with eloxatin, 5-fluoruracil, leukovorin. The patient developed a new local recurrence after successfully surgical treatment of his first relapse. Under these conditions taurolidine treatment appeared to be a final palliative option for our patient since traditional therapeutic modalities failed to control disease progression. In our palliative intravenous study no side effects were observed.

Table 2: Qualitiy of life score (mean) during intravenous TRD-treatment

\begin{tabular}{|c|c|c|}
\hline & Day I & Day 5 \\
\hline Physical & 10.6 & 10.7 \\
\hline Emotional & 4.1 & 4.0 \\
\hline Social & 3.0 & 3.0 \\
\hline Fatigue & 12.2 & 11.8 \\
\hline Pain & 1.0 & 1.0 \\
\hline Dyspnea & 2.1 & 2.2 \\
\hline Global quality of life & 9.0 & 9.1 \\
\hline
\end{tabular}


After an intravenous therapy, leading to partial remission, the chemotherapy was terminated following the patient's wish. After a successfully radiofrequency ablation of one liver metastasis (S8) the taurolidine therapy was initiated again leading to a radiological (CT) stable disease. The patient died due to a postoperative complication after urological surgery, but post mortem histology of esophago-jejunal anastomosis and liver revealed no tumor at all. This finding was a really surprising one as it seems that taurolidine had caused a complete remission of the gastointestinal tumor (gastric cancer). This result could be only poorly expected with the conventional chemotherapy. There are literature reports that the agent might be effective in the treatment of other tumors as well. Taurolidine seems to have a multimodal efficacy on different malignancies both in human as well as in animal models. Stendel et al, noticed a partial remission of glioblastoma in two patients [23] which were treated with $20 \mathrm{~g}$ taurolidine per day. The substance has been found to exert a direct and selective effect on glial and neuronal brain tumor cells via presently unknown apoptotic pathways [13]. The suppression of tumor growth could be also explained by intracellular effects causing apoptosis [24] presumably by a mitochondria cytochrome c-dependent apoptotic mechanism [11], reduction of the TNFa and VEGF production on malignant tumor cells [25]. Moreover, recently the suppression of protein biosynthesis was found to lead to cell death in malignancies (IC50 approximately $1.4 \mathrm{mM}$ ) [15]. For example for abdominal lavage $0.5 \%$ taurolidine corresponding to approximately $16 \mathrm{mM}$ are used for short time periods such as 2 hours in our clinic. The current patient received a weight-adjusted treatment (reaching up to $1.6 \mathrm{mM}$ blood concentration) opposite to the glioblastoma patients (20 g each) whose therapy was successful. On the contrary, urothelial carcinoma was not affected. These findings might suggest that concentrations used exert dissimilar antineoplastic effects on different entities. The specific anti-tumor consequences have been assessed to purge tumor cells from chimeric mixtures of bone marrow resulting in a selective and complete elimination of viable cancer cells [26]. Taurolidine has been described to have immune modulating effects [24]. Therefore, peripheral leukocyte counts were analyzed to determine leukopenia which is a common side effect of other chemotherapeutic agents. In the current case leukopoesis and thrombopoesis, which occurs in the bone marrow, were not impaired. This fact should be considered as a major advantage of this agent compared to the devastating cytotoxic effects of conventional chemotherapeutics and it might implicate that the intravenous use might be safe even in immune suppressed patients in advanced tumor stages. Moreover, these findings will become more evident by using higher concentrations for other malignant entities. It is obvious that toxicity can not be assessed only in one patient. A new phase III clinical study about influence of taurolidine on gastrointestinal tumor recurrences in over 25 patients is currently being conducted in our Department. Results are expected with great interest, but first unpublished data show a favorable outcome as far as toxicity is concerned.

Taking the current findings together, the antineoplastic efficacy seems to be a mixture of the mentioned mechanisms. The intravenous 5 -day-therapy of $2 \%$ taurolidine is safe and anti-tumorigenic in the advanced gastric cancer. On the contrary, urothelial carcinoma was not affected. A clinical phase clinical III study has been set up to evaluate the benefit of the intravenous efficacy on progressive tumor growth. The results are expected with great interest.

\section{Competing interests}

The substance (taurolidine) which was administered during this phase III clinical study was supplied by the Geistlich Pharma AG, Wohlhusen, Switzerland.

\section{Authors' contributions}

C. B. monitored the patient, collected and interpreted the data, and wrote the manuscript. G. W. collected the follow-up data and did the statistical analysis. P. R. was responsible for radiology diagnostics. C. M. was involved in drafting the manuscript and participated in the sequence alignment. C. A. J. contributed to the idea, designed the protocol, conceived and coordinated the study, gathered the data, and reviewed the manuscript. C. A. J. and C. B. are guarantors of the report.

\section{Acknowledgements}

Written consent was obtained from the patient for publication of this case report. We thank Professor Dr. J. M. Mueller, the Director of the Department of Surgery, for continuous support.

\section{References}

I. Maeta M, Yamashiro H, Saito H, Katano K, Kondo A, Tsujitani S, Ikeguchi $M$, Kaibara N: A prospective pilot study of extended (D3) and superextended para-aortic lymphadenectomy (D4) in patients with T3 or T4 gastric cancer managed by total gastrectomy. Surgery 1999, I 25:325-331.

2. Cuschieri A, Fayers P, Fielding J, Craven J, Bancewicz J, Joypaul V, Cook P: Postoperative morbidity and mortality after DI and D2 resections for gastric cancer: preliminary results of the MRC randomised controlled surgical trial.The Surgical Cooperative Group. Lancet 1996, 347:995-999.

3. Wanebo HJ, Kennedy BJ, Winchester DP, Fremgen A, Stewart AK: Gastric carcinoma: does lymph node dissection alter survival? J Am Coll Surg 1996, I 83:616-624.

4. Bonenkamp JJ, Songun I, Hermans J, Sasako M, Welvaart K, Plukker JT, van Elk P, Obertop H, Gouma DJ, Taat CW, .: Randomised comparison of morbidity after DI and D2 dissection for gastric cancer in 996 Dutch patients. Lancet 1995, 345:745-748.

5. Klein HO: [Neo-adjuvants, adjuvants and palliative therapy for gastric carcinoma]. Schweiz Rundsch Med Prax 1998, 87:45|-454.

6. Jacobi CA, Peter FJ, Wenger FA, Ordemann J, Muller JM: New therapeutic strategies to avoid intra- and extraperitoneal metastases during laparoscopy: results of a tumor model in the rat. Dig Surg 1999, 16:393-399. 
7. McCourt M, Wang JH, Sookhai S, Redmond HP: Taurolidine inhibits tumor cell growth in vitro and in vivo. Ann Surg Oncol 2000, 7:685-691.

8. Braumann C, Ordemann J, Wildbrett $P$, Jacobi CA: Influence of intraperitoneal and systemic application of taurolidine and taurolidine/heparin during laparoscopy on intraperitoneal and subcutaneous tumour growth in rats. Clin Exp Metastasis 2000, I 8:547-552.

9. Braumann C, Ordemann J, Kilian M, Wenger FA, Jacobi CA: Local and systemic chemotherapy with taurolidine and taurolidine/heparin in colon cancer-bearing rats undergoing laparotomy. Clin Exp Metastasis 2003, 20:387-394.

10. Calabresi P, Goulette FA, Darnowski JW: Taurolidine: cytotoxic and mechanistic evaluation of a novel antineoplastic agent. Cancer Res 2001, 61:6816-6821.

II. Han Z, Ribbizi I, Pantazis P, Wyche J, Darnowski J, Calabresi P: The antibacterial drug taurolidine induces apoptosis by a mitochondrial cytochrome c-dependent mechanism. Anticancer Res 2002, 22:1959-1964.

12. Shrayer DP, Lukoff H, King T, Calabresi P: The effect of Taurolidine on adherent and floating subpopulations of melanoma cells. Anticancer Drugs 2003, 14:295-303.

13. Stendel R, Stoltenburg-Didinger G, Al Keikh CL, Wattrodt M, Brock $M$ : The effect of taurolidine on brain tumor cells. Anticancer Res 2002, 22:809-814.

14. Van Antwerp DJ, Martin SJ, Verma IM, Green DR: Inhibition of TNF-induced apoptosis by NF-kappa B. Trends Cell Biol 1998, 8:|07-III.

15. Braumann C, Henke W, Jacobi CA, Dubiel W: The tumor-suppressive reagent taurolidine is an inhibitor of protein biosynthesis. Int J Cancer 2004, I I 2:225-230.

16. Aaronson NK, Ahmedzai S, Bergman B, Bullinger M, Cull A, Duez NJ, Filiberti A, Flechtner H, Fleishman SB, de Haes JC, .: The European Organization for Research and Treatment of Cancer QLQC30: a quality-of-life instrument for use in international clinical trials in oncology. J Natl Cancer Inst 1993, 85:365-376.

17. de Manzoni G, Verlato G, Guglielmi A, Laterza E, Genna M, Cordiano $C$ : Prognostic significance of lymph node dissection in gastric cancer. Br J Surg 1996, 83:1604-1607.

18. Siewert JR, Bottcher K, Roder JD, Busch R, Hermanek P, Meyer HJ: Prognostic relevance of systematic lymph node dissection in gastric carcinoma. German Gastric Carcinoma Study Group. Br J Surg 1993, 80:1015-1018.

19. Hermans J, Bonenkamp JJ, Boon MC, Bunt AM, Ohyama S, Sasako M, Van de Velde Cl: Adjuvant therapy after curative resection for gastric cancer: meta-analysis of randomized trials. J Clin Oncol 1993, I I:| 44 I-| 447.

20. Nakajima T: Review of adjuvant chemotherapy for gastric cancer. World J Surg 1995, 19:570-574.

21. Darnowski JW, Goulette FA, Cousens LP, Chatterjee D, Calabresi P: Mechanistic and antineoplastic evaluation of taurolidine in the DUI45 model of human prostate cancer. Cancer Chemother Pharmacol 2004, 54:249-258.

22. Nici L, Monfils B, Calabresi P: The effects of taurolidine, a novel antineoplastic agent, on human malignant mesothelioma. Clin Cancer Res 2004, 10:7655-766I.

23. Stendel R, Picht T, Schilling A, Heidenreich J, Loddenkemper C, Janisch W, Brock M: Treatment of glioblastoma with intravenous taurolidine. First clinical experience. Anticancer Res 2004, 24: | |43-I |47.

24. Yanagisawa M, Imai H, Fukushima $Y$, Yasuda $T$, Miura $A B$, Nakamoto $Y$ : Effects of tumour necrosis factor alpha and interleukin I beta on the proliferation of cultured glomerular epithelial cells. Virchows Arch 1994, 424:581-586.

25. Torisu H, Ono M, Kiryu H, Furue M, Ohmoto Y, Nakayama J, Nishioka $Y$, Sone S, Kuwano M: Macrophage infiltration correlates with tumor stage and angiogenesis in human malignant melanoma: possible involvement of TNFalpha and IL-I alpha. Int J Cancer 2000, 85: 182-188.

26. Ribizzi I, Darnowski JW, Goulette FA, Akhtar MS, Chatterjee D, Calabresi P: Taurolidine: preclinical evaluation of a novel, highly selective, agent for bone marrow purging. Bone Marrow Transplant 2002, 29:313-319.
Publish with Biomed Central and every scientist can read your work free of charge

"BioMed Central will be the most significant development for disseminating the results of biomedical research in our lifetime. "

Sir Paul Nurse, Cancer Research UK

Your research papers will be:

- available free of charge to the entire biomedical community

- peer reviewed and published immediately upon acceptance

- cited in PubMed and archived on PubMed Central

- yours - you keep the copyright

Submit your manuscript here:

http://www.biomedcentral.com/info/publishing_adv.asp
BiolMedcentral 